\title{
Dilators Adapted to Continuous Suction System for Tapping and Pedicle Screw Placement for Minimally Invasive Lumbosarcal Fixation
}

\author{
Yunfen $\mathbf{W u}^{*}$ \\ Department of Science of Health, University of Cantabria, Spain
}

Received: 眥 August 20, 2018; Published: 制 September 04, 2018

*Corresponding author: Yunfen Wu, Department of Intraoperative Neurophysiology, University of Cantabria, Spain

\begin{abstract}
Background: Stimulus-evoked EMG improves the safety during pedicle screw placement in the lumbosacral spine. The reported reliability of stimulus-evoked EMG in the detection of pedicle breaches in minimally invasive lumbosacral fixation has been variable. Besides the influence from different physiopathological conditions, current shunting may also contribute in this variable reliability due to the small incision during minimally invasive surgery. Using a traditional suction system, continues suction is not viable during tapping and pedicle screw placement in minimally invasive surgery.
\end{abstract}

Objective: The author proposes a new design of non-metallic dilators adapted to continuous suction during tapping and pedicle screw placement in minimally invasive surgery with the aim of minimizing the possible current shunting and optimizing the stimulation accuracy.

Materials and Methods: The proposed nonmetallic dilators have 2 canals. The larger canal would be used for the passage of the tap and the pedicle screw attached to the screwdriver. The lumen of the smaller canal would be connected to the connecting tubing attached to a reservoir with vacuum pressure. After a small skin incision was made, each dilator was placed into a piece of porcine meat. The main canal of both dilators was partially sealed to simulate the passage of the instrumentation devices. Porcine blood was continuously injected into the created cavity to simulate the concurrent bleeding.

Results/Observations: By applying a suction pressure set to $15 \mathrm{cmHg}$, the aspirated porcine blood was seen through the clear tubing.

Conclusion: In this experimental set-up, the proposed dilators showed an excellent operation while realizing that further electrophysiological experiment is necessary to confirm clinical relevance.

Keywords: EMG; Dilator; Lumbar Fixation; Pedicle Screw; Percutaneous; Continuous Suction

\section{Introduction}

Pedicle screw instrumentation for the stabilization and correction of the lumbosacral spine has been continuously growing in popularity [1,2]. Minimally invasive techniques offer benefits including reduced tissue injury and blood loss allowing less postoperative pain and a quicker recovery [1]. However, the risk of neurological injury is greater in minimally invasive procedures due to the lack of a direct visualization of the anatomical landmarks and inspection [1,2]. Stimulus-evoked Electromyography (EMG) monitoring improves the safety during the pedicle screw placement in the lumbosacral spine. Nevertheless, the reported reliability of stimulus-evoked EMG in the detection of pedicle breaches in minimally invasive lumbosacral fixation surgery has been variable [1-4]. Besides the influence from different physiopathological conditions [5-7], current shunting may also contribute to this variable reliability $[8,9]$. Continuous suction during tapping and pedicle screw placement in minimally invasive surgery is not viable with the use of a traditional suction system. The increased risk of current shunting may alter the stimulation thresholds and the identification of potential breaches occasionally and leading false negative findings even when high stimulation thresholds are used [4]. The author proposes a concept of modified non-metallic dilators adapted to continuous suction during tapping and pedicle screw placement with the aim of minimizing the possible current shunting and consequently optimizing the stimulation accuracy.

\section{Method and Materials}

\section{Design of the Proposed Dilators}

The proposed non-metallic dilators are constructed by using the dimensions of the existing dilators of the Stryker ES2® Spinal 
System. The proposed dilators, in addition to the main canal of each dilator being used for the passage of the tapscrew and the pedicle screw attached to the screwdriver, have a $2 \mathrm{~mm}$ diameter canal which would be connected to a conventional vacuum pump system. The location of the connecting portion allows for the proper handling for insertion of the dilators. The suction canal inlet is located $1 \mathrm{~mm}$ above the bottom of the main canal of the dilators (Figure 1). The prototype dilators for the experiment were manufactured with a commercial grade $3 \mathrm{~d}$ printer using plastic material with 16 micron layer precision (Figure 2).
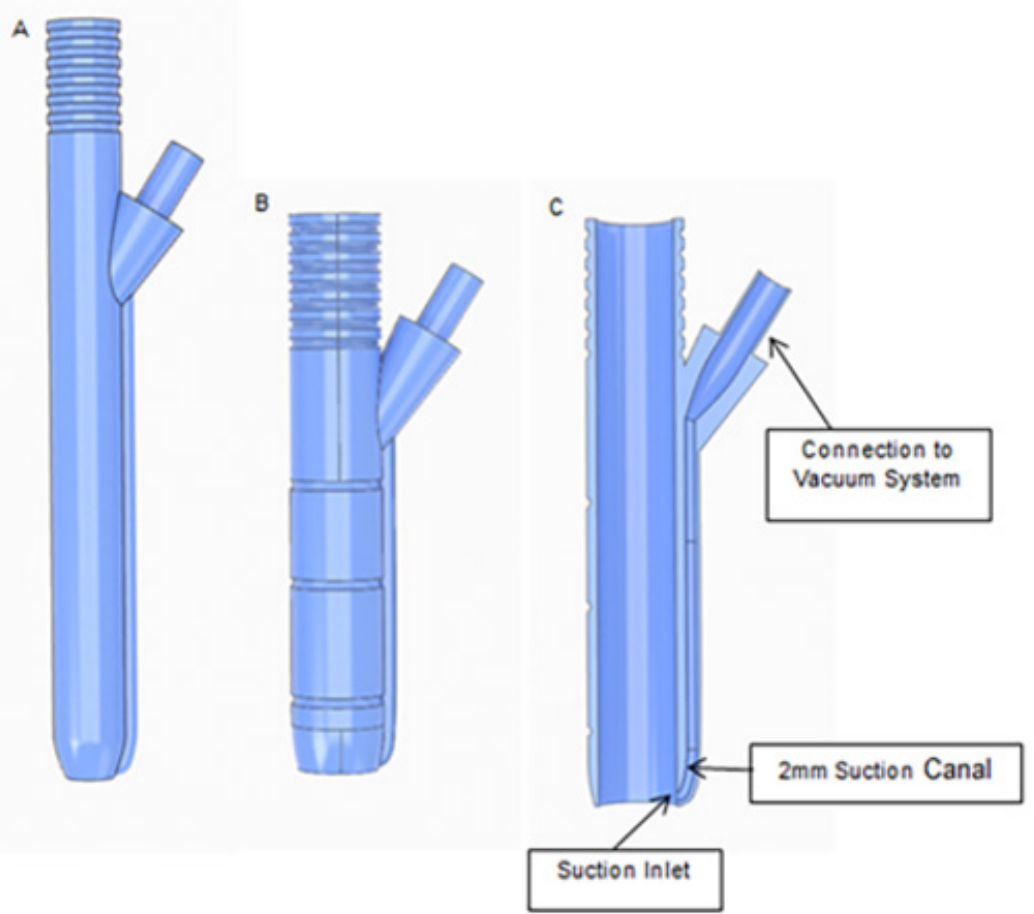

Figure 1: Proposed nonmetallic dilator for the passage of the tapscrew (1A). Proposed nonmetallic dilator for the passage of the pedicle screw attached to the screwdriver (1B) and cross section view (1C).

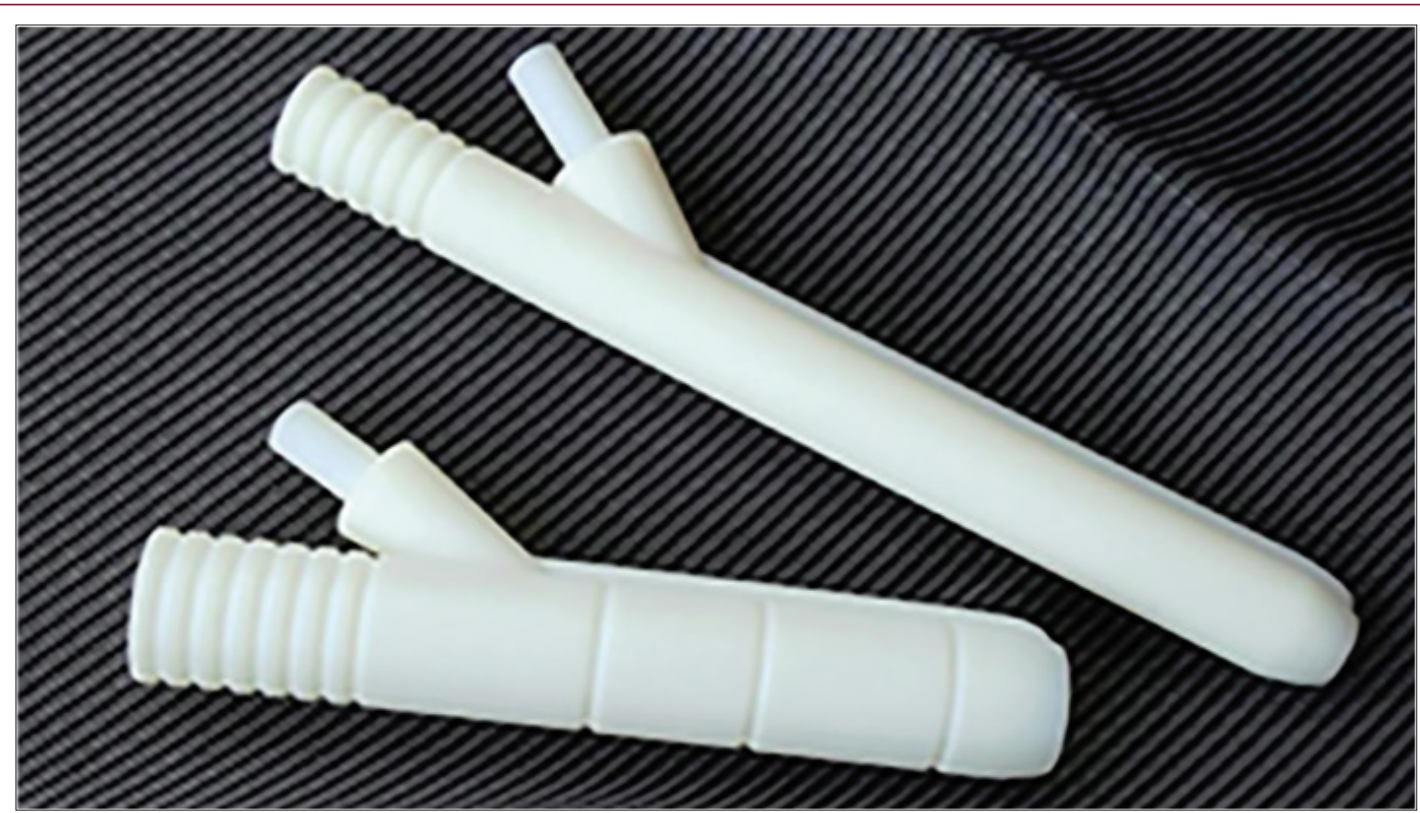

Figure 2: 3D Printed plastic prototypes.

Porcine blood has been reported to be an appropriate alternative to human blood regarding to their similar physical parameters (surface tension, viscosity and density) [10]. In this experiment, fresh and refrigerated porcine blood acquired from a supermarket was used to simulate human blood.

\section{Protocol 1}

A small skin incision was made in a piece of porcine meat. The proposed nonmetallic dilator for the passage of the tapscrew was placed into the porcine meat to create a muscular tunnel and a 
minimum cavity. The standard suction tubing was connected to the small lumen of the proposed dilator. A screw driver that simulates a surgical tapscrew was placed into the big canal causing a partial seal. The suction machine was turned "ON" while porcine blood was continuously injected in the created cavity to simulate the concurrent bleeding with tapping (Video 1).

Video 1: 0:01 - Incision; 0:08 - Placement of the dilator; 0:20 - Connection of the suction to the dilator; 0:46 - Placement of the screw driver; 0:52 - Injection of porcine blood.

\section{Protocol 2}

The previous incision has been extended for the placement of a larger nonmetallic dilator. This dilator has been proposed for the passage of the pedicle screw attached to the screwdriver. The small lumen of the dilator was connected to the standard tubbing. A circular solid stick that simulates the pedicle screw attached to the screwdriver was placed into the larger canal causing a partial seal. The suction machine was turned "ON" while porcine blood was continuously injected into the created cavity to simulate the concurrent bleeding with the pedicle screw placement (Video 2).

Video 2: 0:04 - Extension of the previous incision; 0:12 Placement of the dilator; 0:21 - Connection of the suction to the dilator; 0:31 - Placement of the circular solid stick; 0:52 - Injection of porcine blood.

\section{Results}

\section{Protocol 1 and protocol 2}

Numerous pressure tests by means of regulator adjustment were conducted to determine optimal pressure for the suction of the blood. By applying suction pressure set to $15 \mathrm{cmHg}$, the aspirated blood was seen through the clear tubing.

\section{Discussion}

During minimally invasive pedicle screw placement, there is a lack of a complete reliability in identifying misplaced screws based on intraoperative imaging $[11,12]$. Stimulus-evoked EMG with testing of the instrumentation devices is becoming an important monitoring tool that provides a functional assessment of the structural integrity of the pedicle. This monitoring technique can detect unidentified mal positioned pedicle screws by using imaging techniques and help to prevent suboptimal screw trajectories $[2,13,14]$. Nevertheless, the reported reliability of stimulusevoked EMG monitoring in the detection of pedicle breaches during minimally invasive transpedicular screw placement for lumbosacral fixation has been variable [1-4]. A variation of physiopathological conditions (i.e. the bone mineral density and; chronic nerve compression with axonotmesis and axon loss) and other factors such as current shunting should be considered for an accurate judgment of the stimulation threshold [5-9].

In minimally invasive procedures, the initial pilot trajectory is usually created by the pedicle access needle. The proper trajectory of the pedicle access needle can be confirmed by the imaging and the stimulation threshold. Supplementary testing of the tapscrew and the pedicle screw provides additional benefit due to their larger intraosseous extension when compared with only the pedicle access needle testing. Persistent stimulation of the tapscrew and the pedicle screw with the use of a non-metallic dilator may provide immediate feedback of an early inappropriate trajectory [15]. During stimulation, suction of wound fluids and retraction from soft tissue contact are always favorable for an accurate evaluation of "pilot" hole/screw thresholds. However, in regards to the small incision, isolation may still have some limitations even with use of a non-metallic dilator. Bleeding is concurrent with tapping and pedicle screw placement due to the intraosseous advance of the tap and the screw. Occasional excessive wound fluids surrounding the tapscrew and the pedicle screw can erroneously elevate the stimulation thresholds due to current shunting, thus leading to false-negative findings even when high stimulation thresholds are used $[4,8,9]$. During tapping and pedicle screw placement in minimally invasive surgery, the use of a traditional suction system does not allow suction of the wound fluids during the implantation of the instrumentation devices.

The author introduces a new design of dilators adapted to continuous suction during tapping and pedicle screw placement. These dilators should be made of a non-metallic material that allows an atraumatic placement and behaves as an insulating sleeve (soft-tissue barrier) over the instrumentation devices. The primary difference from the commercially available dilators consists of the number of dilator canals and the adaptability to the existent vacuum delivery system. Each proposed dilator has two canals. The resistant construction avoids collapse during suction. The main and the larger canal would be used for the passage of the tapscrew and the pedicle screw attached to the screwdriver. The diameter of this larger canal should be adapted to the diameter of the tapscrew and the pedicle screw attached to the screwdriver. The secondary smaller canal is large enough to evacuate the particles of tissue or blood clots. The end portion (lumen) of this 
small canal allows connection to the standard connecting tubing. The connecting tubing will be connected to a collection canister with vacuum pressure.

The length and the inner diameter of the tubing, and the internal diameter of the tubing connectors are the most important factors affecting the resistance of the suction equipment. Ideally, the tubing and connectors should be as large in diameter (minimum 0.25 in or $6 \mathrm{~mm}$ ) and the tubing as short as possible [16]. The proper diameter ( $2 \mathrm{~mm}$ ) of the small canal of both proposed dilators connected to the suction system minimize the negative impact in the resistance increment. In our experiment, tubing of $6 \mathrm{~mm}$ in diameter and with a length of $6.1 \mathrm{~m}$ was used. The long length of the tubing increases the challenge in the functioning of the suction but demonstrates the efficacy of both proposed dilators even when long length of tubing is required to be used in the surgery.

The author believes, with the suction of wound fluids from the simulation field, the accuracy of the stimulus-evoked EMG monitoring would be optimized during the testing of the tapscrew and the pedicle screw in minimally invasive surgery. On the other hand, a small diameter of the additional canal of the proposed dilators conserves the concept of minimally invasive procedure with a reduced tissue injury. The present paper aims to introduce a concept of modified dilators. Further research on clinical relevance of wound fluids suction is needed to improve the technical aspects of the stimulus-evoked EMG as a monitoring method in minimally invasive pedicle screw placement.

\section{Conclusion}

In this experimental set-up, the proposed dilators showed an excellent operation. The author considers with the use of nonmetallic dilators, adapted to continuous suction during tapping and pedicle screw placement may minimize the possible current shunting and consequently optimize the accuracy of stimulusevoked EMG monitoring. Further research is necessary in showing the efficacy of the proposed system with clinical trials.

\section{References}

1. Ozgur BM, Berta S, Khiatani V, Taylor WR (2006) Automated intraoperative EMG testing during percutaneous pedicle screw placement. Spine J 6(6): 708-713.

ISSN: 2574-1241

DOI: 10.26717/BJSTR.2018.08.001686

Yunfen Wu. Biomed J Sci \& Tech Res

This work is licensed under Creative

Commons Attribution 4.0 License

Submission Link: https://biomedres.us/submit-manuscript.php
2. Bindal RK, Ghosh S (2007) Intraoperative electromyography monitoring in minimally invasive transforaminal lumbar inlerbody fusion. J Neurosurg Spine 6(2): 126-132.

3. Wood M, Mannion R (2011) A comparison of CT-based navigation techniques for minimally invasive lumbar pedicle screw placement. J Spinal Disord Tech 24(1): E1-E5.

4. Wang MY, Pineiro G, Mummaneni PV (2010) Stimulus-evoked electromyography testing of percutaneous pedicle screws for the detection of pedicle breaches: A clinical study of 409 screws in 93 patients. J Neurosurg Spine 13(5): 600-605.

5. Holland NR, Lukaczyk TA, Riley LH 3rd, Kostuik JP (1998) Higher electrical stimulus intensities are required to activate chronically compressed nerve roots. Implications for intraoperative electromyographic pedicle screw testing. Spine 23(2): 224-227.

6. Lu WW, Zheng Y, Holmes A (2000) Bone mineral density variations along the lumbosacral spine. Clin Orthop Relat Res 378: 255-263.

7. Dickerman RD, Guyer R (2006) Letter to the Editor: Intraoperative electromyography for pedicle screws: Technique is the key! J Spinal Dis Tech19: 463.

8. Isley MR, Zhang XF, Balzer JR, Leppanen RE (2012) Current Trends in Pedicle Screw Stimulation Techniques: Lumbosacral, Thoracic, and Cervical Levels. Neurodiagn J 52(2): 100-175.

9. Wu Y, Vázquez Barquero A (2017) Stimulus-evoked EMG monitoring during minimally invasive transpedicular implantation of screws in the lumbosacral spine - Threshold value, methodology and clinical effectiveness. World Neurosurg 98: 146-151.

10. Raymond MA, Smith ER, Liesegang J (1996) The physical properties of blood--forensic considerations. Sci Justice 36(3): 153-160.

11. Yoo JU, Ghanayem A, Petersilge C, Lewin J (1997) Accuracy of using computed tomography to identify pedicle screw placement in cadaveric human lumbar spine. Spine 22(22): 2668-2671.

12. Kim YJ, Lenke LG, Bridwell KH, Cho YS, Riew KD (2004) Free hand pedicle screw placement in the thoracic spine: Is it safe? Spine 29(3): 333-342.

13. Calancie B, Madsen P, Lebwohl N (1994) Stimulus-evoked EMG monitoring during transpedicular lumbosacral spine instrumentation. Initial clinical results. Spine 19(24): 2780-2786.

14. Toleikis JR, Skelly JP, Carlvin AO, Toleikis SC, Bernard TN, et al. (2000) The usefulness of electrical stimulation for assessing pedicle screw placements. J Spinal Disord 13(4): 283-289.

15. Yunfen Wu, Alfonso Vázquez Barquero, Dhiraj R Jeyanandarajan (2018) Can Pedicle Screws be Tested During Minimally Invasive Lumbosacral Fixation? Biomedical Journal of Scientific \& Technical Research 8(1).

16. Lam B, Pursley D (2014) The Principles of Vacuum and Clinical Application in the Hospital Environment. ( $3^{\text {rd }}$ edn); Springfield: Kenney

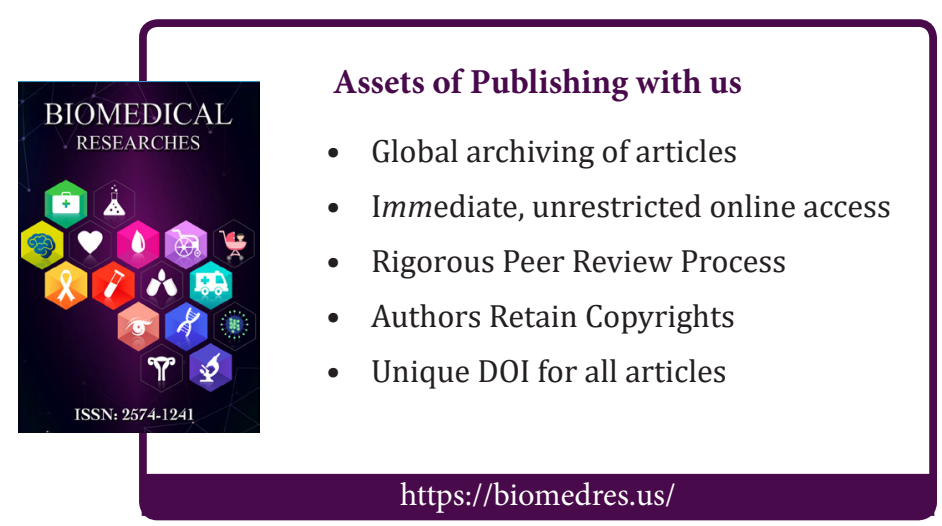

